\title{
Experimental realization of a programmable quantum-state discriminator and a phase-covariant quantum multimeter
}

\author{
Jan Soubusta, ${ }^{1,2}$ Antonín Černoch, ${ }^{2}$ Jaromír Fiurášek, ${ }^{3,2}$ and Miloslav Dušek ${ }^{2}$ \\ ${ }^{1}$ Joint Laboratory of Optics of Palacky University and Institute of Physics of Academy of Sciences of the Czech Republic, \\ 17. listopadu 50A, 77200 Olomouc, Czech Republic \\ ${ }^{2}$ Department of Optics, Palacký University, 17. listopadu 50, 77200 Olomouc, Czech Republic \\ ${ }^{3}$ QUIC, Ecole Polytechnique, CP 165, Université Libre de Bruxelles, 1050 Bruxelles, Belgium
}

(Dated: December 2, 2018)

\begin{abstract}
We present an optical implementation of two programmable quantum measurement devices. The first one serves for unambiguous discrimination of two nonorthogonal states of a qubit. The particular pair of states to be discriminated is specified by the quantum state of a program qubit. The second device can perform von Neumann measurements on a single qubit in any basis located on the equator of the Bloch sphere. Again, the basis is selected by the state of a program qubit. In both cases the data and program qubits are represented by polarization states of photons. The experimental apparatus exploits the fact that two Bell states can be distinguished solely by means of linear optics. The outcome corresponding to the remaining two Bell states represents an inconclusive result.

PACS numbers: 03.65.-w, 03.67.-a, 42.50.Dv
\end{abstract}

\section{INTRODUCTION}

Quantum measurements are inevitable parts of all quantum devices 1, 2, 3, 4]. In many situations, the choice of a particular measurement depends on the task to be performed. For instance, in the case of quantumstate discrimination the choice of the generalized measurement is given by the specific pair of states that are supposed to be discriminated. Recently universal (multipurpose) quantum measurement devices, "quantum multimeters", were introduced and discussed in several papers $[5,6,67,8,9]$. Their key property is the possibility to control the choice of the measurement by a quantum state of the program register, which could be in principle unknown. The quantum states of a program register corresponding to different measurements are allowed to be mutually non-orthogonal.

In this paper, we report on the experimental realization of a programmable quantum-state discriminator (Section III) and a phase-covariant multimeter (Section IIII). Both programmable detectors involve two qubits, one data qubit and one program qubit. In our optical implementation, the qubits are encoded into polarization states of single photons and the required photon pairs are generated by means of spontaneous parametric downconversion. The experiment exploits the fact that a partial Bell measurement on polarization states of two photons can be accomplished with linear optics, namely a balanced beamsplitter and two polarizing beamsplitters, followed by photodetectors 10, 11, 12, 13, 14.

\section{PROGRAMMABLE QUANTUM-STATE DISCRIMINATOR}

\section{A. Theory}

A general unknown quantum state cannot be determined completely by a measurement performed on a single copy of the system. But the situation is different if $a$ priori knowledge is available [1, 2, 3] - e.g., if one works only with states from a certain discrete set. Even quantum states that are mutually non-orthogonal can be distinguished with a certain probability provided they are linearly independent (for a review see Ref. 15|). There are, in fact, two different optimal strategies 16]: First, the strategy that determines the state with the minimum probability for the error [1, 2] and, second, unambiguous or error-free discrimination (the measurement result never wrongly identifies a state) that allows the possibility of an inconclusive result (with a minimal probability in the optimal case) 17, 18, 19, 20, 21]. We will concentrate our attention to the unambiguous state discrimination. It has been first investigated by Ivanovic 17] for the case of two equally probable non-orthogonal states. Peres 19] solved the problem of discrimination of two states in a formulation with POVM measurement. Later Jaeger and Shimony 20] extended the solution to arbitrary a priori probabilities. Chefles and Barnett 21] have generalized Peres's solution to an arbitrary number of equally probable states which are related by a symmetry transformation. Unambiguous state discrimination was already realized experimentally. The first experiment, designed for the discrimination of two linearly polarized states of light, were done by Huttner et al. 22. The interest in the quantum state discrimination is not only "academic" - unambiguous state discrimination can be used, e.g., as an efficient attack in quantum cryptography [23].

Let us now suppose that we want to discriminate un- 

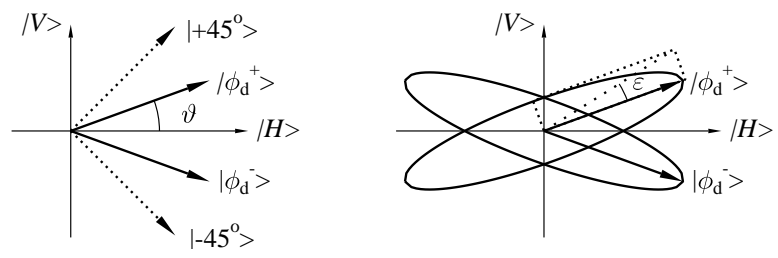

FIG. 1: Polarization of the "data" photon: left panel - linear polarizations, right panel - elliptical polarizations.

ambiguously between two non-orthogonal states. However, we would like to have a possibility to "switch" the apparatus in order to be able to work with several different pairs of states. The switching should be realized by preparing a program register of the programmable discriminator in different program states. The program state thus specifies which pair of quantum states is discriminated by the device. This problem was investigated theoretically in Ref. [5].

Let us have two (non-orthogonal) input states of a qubit that should be discriminated:

$$
\left|\phi_{\mathrm{d}}^{ \pm}\right\rangle=a\left|H_{\mathrm{d}}\right\rangle \pm b\left|V_{\mathrm{d}}\right\rangle,
$$

where $\left|H_{\mathrm{d}}\right\rangle$ and $\left|V_{\mathrm{d}}\right\rangle$ represent two "logical" levels of a qubit, in particular horizontal and vertical linear polarizations of a photon; subscript d stays for "data". Note that these states are supposed to be symmetrically located around the state $\left|H_{\mathrm{d}}\right\rangle$, as depicted in Fig. 1] In addition let us have a program qubit in a state (index $\mathrm{p}$ denotes "program"):

$$
\left|\phi_{\mathrm{p}}\right\rangle=a^{\prime}\left|H_{\mathrm{p}}\right\rangle+b^{\prime}\left|V_{\mathrm{p}}\right\rangle .
$$

Choosing properly the state of a program qubit and performing a suitable joint measurement on the data and program qubits together one can unambiguously discriminate any two states of the data qubit that are in agreement with the program.

Let us suppose that the state of the program qubit is equal to one of the two states that shall be error-free discriminated. In particular, let

$$
\left|\phi_{\mathrm{p}}\right\rangle=a\left|H_{\mathrm{p}}\right\rangle+b\left|V_{\mathrm{p}}\right\rangle .
$$

Then the total state of the data and program qubit reads

$$
\begin{gathered}
\left|\phi_{\mathrm{d}}^{ \pm}\right\rangle \otimes\left|\phi_{\mathrm{p}}\right\rangle= \\
\sqrt{2}\left[\frac{a^{2} \pm b^{2}}{2}\left|\Phi^{+}\right\rangle+\frac{a^{2} \mp b^{2}}{2}\left|\Phi^{-}\right\rangle+a b\left|\Psi^{ \pm}\right\rangle\right],
\end{gathered}
$$

where

$$
\begin{aligned}
& \left|\Psi^{ \pm}\right\rangle=\frac{1}{\sqrt{2}}\left(\left|H_{\mathrm{d}}\right\rangle\left|V_{\mathrm{p}}\right\rangle \pm\left|V_{\mathrm{d}}\right\rangle\left|H_{\mathrm{p}}\right\rangle\right), \\
& \left|\Phi^{ \pm}\right\rangle=\frac{1}{\sqrt{2}}\left(\left|H_{\mathrm{d}}\right\rangle\left|H_{\mathrm{p}}\right\rangle \pm\left|V_{\mathrm{d}}\right\rangle\left|V_{\mathrm{p}}\right\rangle\right)
\end{aligned}
$$

are the Bell states. Clearly, if we were able to detect Bell states $\left|\Psi^{+}\right\rangle$and $\left|\Psi^{-}\right\rangle$we could unambiguously discriminate states $\left|\phi_{\mathrm{d}}^{+}\right\rangle$and $\left|\phi_{\mathrm{d}}^{-}\right\rangle$.
The probability of successful discrimination would be

$$
p=2|a b|^{2}=2\left(|a|^{2}-|a|^{4}\right) .
$$

This probability of success is not optimal in general. As shown in Refs. 17, 18, 19] the optimal probability of successful discrimination $p_{\mathrm{opt}}=1-\left|\left\langle\phi_{\mathrm{d}}^{+} \mid \phi_{\mathrm{d}}^{-}\right\rangle\right|=$ $1-\left.|2| a\right|^{2}-1 \mid$. In fact, the probability (6) corresponds to a "quasi-classical" discrimination. By a quasi-classical approach we mean a probabilistic measurement when one randomly selects [25] the projective measurement in one of two bases that both span the two-dimensional space containing both non-orthogonal states of interest (1). One basis consists of the state $\left|\phi_{\mathrm{d}}^{+}\right\rangle$and its orthogonal complement $\left|\phi_{\perp \mathrm{d}}^{+}\right\rangle$. If one finds the result corresponding to $\left|\phi_{\perp d}^{+}\right\rangle$he/she can be sure that the state $\left|\phi_{d}^{+}\right\rangle$was not present. Analogously, the other basis consists of the state $\left|\phi_{\mathrm{d}}^{-}\right\rangle$and its orthogonal complement.

To get a higher probability of success one would have to make a more sophisticated measurement than a simple Bell-state analysis [5]. However, there are two important points concerning the proposed discrimination procedure: First, a partial measurement in the Bell basis can be easily realized by linear optics. Namely, states $\left|\Psi^{+}\right\rangle$and $\left|\Psi^{-}\right\rangle$can be distinguished (the rest of the Bell states corresponds to an inconclusive result anyway). Second, our aim is not to maximize the success probability but to demonstrate experimentally the possibility to control the discrimination process by the quantum state of a program qubit. Even to set the bases for the mentioned quasiclassical discrimination correctly, one needs an infinite number of bits of classical information. Our procedure requires only a single qubit for the same job. This fact reveals the quantum nature of the programming. Even non-orthogonal states of the quantum qubit carry "useful information" for the discrimination process.

\section{B. Experiment}

The scheme of our experimental setup is shown in Fig. 2 A krypton-ion $\mathrm{cw}$ laser $(413.1 \mathrm{~nm}, 95 \mathrm{~mW})$ is used to pump a 10-mm-long $\mathrm{LiO}_{3}$ nonlinear crystal cut for degenerate type-I parametric downconversion. We exploit the fact that the pairs of photons generated by spontaneous parametric downconversion (SPDC) manifest tight time correlations (i.e., very exact coincidences of detection instants). In our setup the photons produced by SPDC have horizontal linear polarizations. Different polarization states are prepared by means of half-wave and quarter-wave plates (HWP, QWP). The polarization of the signal photon represents the data qubit whilst the polarization of the idler photon serves as the program qubit. The two photons impinge on two input ports of a 50/50 beamsplitter (BS, non-polarizing cube beamsplitter). A scanning mirror is used in one interferometer arm in order to balance the length of both arms, as indicated by an arrow in Fig. 2. The photons reflected and 


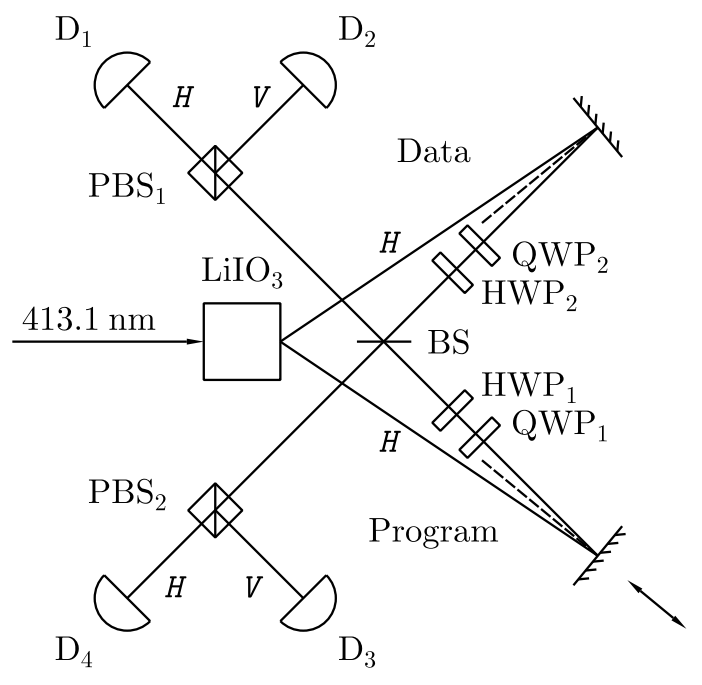

FIG. 2: Experimental setup (BS is a beamsplitter, PBS polarization beamsplitters, D denotes detectors, and HWP and QWP half- and quarter-wave plates, respectively).

transmitted by BS pass polarizing beamsplitters (PBS, polarizing cube beamsplitter) to distinguish horizontal and vertical polarizations. Finally, the beams are filtered by cut-off filters and circular apertures, and coupled into multi-mode optical fibers by lenses. Detectors $\mathrm{D}_{1}, \ldots, \mathrm{D}_{4}$ are Perkin-Elmer single-photon counting modules (employing silicon avalanche photodiodes with quantum efficiency $\eta \approx 50 \%$ and dark counts about $100 \mathrm{~s}^{-1}$ ). The signals from detectors are processed by our home-made 4 -input coincidence module. With this setup, visibilities of Hong-Ou-Mandel dip 24] exceeding $92 \%$ were reached for vertically polarized photons (for other polarizations visibilities were slightly lower). Higher visibilities could not be reached due to the fact that the splitting ratios of the coupler were not exactly 50/50 and due to the imperfections of the wave plates and polarization distortions on mirrors.

As already mentioned our experiment is based on the possibility to detect the two particular Bell states in the Hilbert space of the polarization states of two photons. This task can be done by means of passive linear optical elements, namely by a beamsplitter and two polarization beamsplitters as shown in Fig. 2] 10, 11, 12, 13, 14].

The simplest theoretical model of the beamsplitter leads to the conclusion that if one fetches Bell states at the inputs the only one of them that results in a coincident detection at two different outputs of the beamsplitter is the singlet state $\left|\Psi^{-}\right\rangle$. However, in case of the "real" beam-splitting cube one must take into account that the two photons strike upon a beamsplitter in opposite directions. So, if the mutual phase of the vertical components of the electric-field vectors at the interface plane (we mean the vectors corresponding to the fields coming from the two opposite inputs) is $\varphi$ the mutual

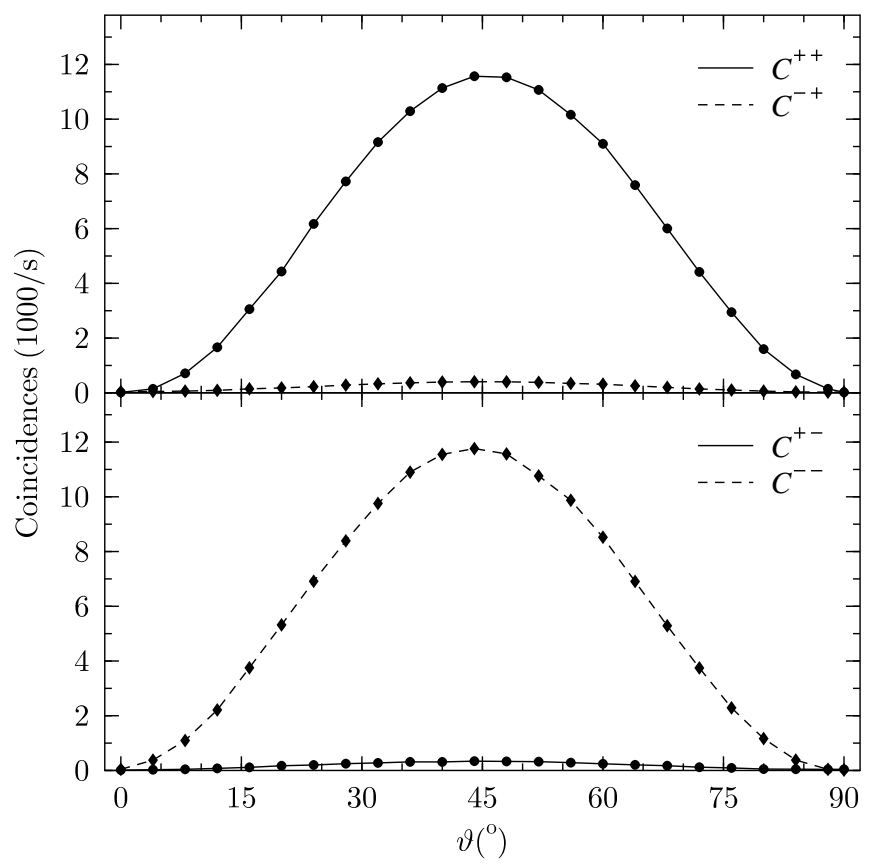

FIG. 3: Detection rates of $\left|\Psi^{+}\right\rangle$(dots, solid line) and $\left|\Psi^{-}\right\rangle$ (diamonds, broken line) for linearly polarized input states. The upper part corresponds to the situation when the data and program states coincide, $\left|\phi_{\mathrm{d}}^{+}\right\rangle \otimes\left|\phi_{\mathrm{p}}\right\rangle$, the lower part to the situation when they are different, $\left|\phi_{\mathrm{d}}^{-}\right\rangle \otimes\left|\phi_{\mathrm{p}}\right\rangle$.

phase of the horizontal components is $\varphi+180^{\circ}$ just for geometrical reasons. It means, if one prepares both photons in the same linear polarizations tilted by $45^{\circ}$ with respect to the plane of incidence the vectors of electric field at the interface plane will oscillate in mutually perpendicular directions. Consequently, now it is the triplet state $\left|\Psi^{+}\right\rangle$that leads to a coincident detection at different outputs.

So, if detectors $\mathrm{D}_{1}$ and $\mathrm{D}_{3}$ or $\mathrm{D}_{2}$ and $\mathrm{D}_{4}$ click together in our setup the state $\left|\Psi^{+}\right\rangle$is detected (at the input of the beamsplitter) - this corresponds to the recognition of state $\left|\phi_{\mathrm{d}}^{+}\right\rangle$. If detectors $\mathrm{D}_{1}$ and $\mathrm{D}_{2}$ or $\mathrm{D}_{3}$ and $\mathrm{D}_{4}$ click in coincidence the state $\left|\Psi^{-}\right\rangle$was "present" in the input - so, $\left|\phi_{\mathrm{d}}^{-}\right\rangle$is detected. If both photons enter the same detector either $\left|\Phi^{+}\right\rangle$or $\left|\Phi^{-}\right\rangle$was "present" in the input - this represents the inconclusive result of the discrimination.

Each data point at presented plots has been derived from 10 one-second measurement periods. The accuracy of polarization-angle settings was better than $\pm 1^{\circ}$.

\section{Results}

We tried to discriminate a broad variety of pairs of elliptically polarized states

$\left|\phi_{\mathrm{d}}^{ \pm}\right\rangle=(x \cos \vartheta+i y \sin \vartheta)\left|H_{\mathrm{d}}\right\rangle \pm(x \sin \vartheta-i y \cos \vartheta)\left|V_{\mathrm{d}}\right\rangle$, 


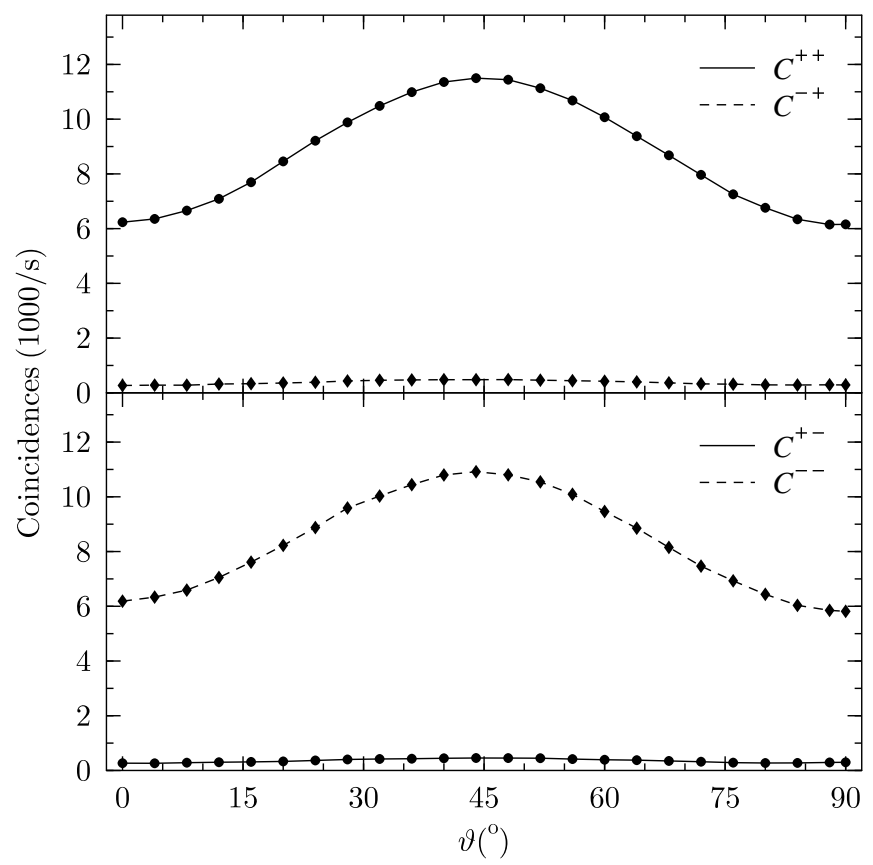

FIG. 4: Detection rates of $\left|\Psi^{+}\right\rangle$(dots, solid line) and $\left|\Psi^{-}\right\rangle$ (diamonds, broken line) for elliptically polarized input states, $\varepsilon=24^{\circ}$. The upper part corresponds to the situation when the data and program states coincide, $\left|\phi_{\mathrm{d}}^{+}\right\rangle \otimes\left|\phi_{\mathrm{p}}\right\rangle$, the lower part to the situation when they are different, $\left|\phi_{\mathrm{d}}^{-}\right\rangle \otimes\left|\phi_{\mathrm{p}}\right\rangle$.

where $x$ and $y$ are the half-axes of the polarization ellipse and $\vartheta$ is the angle between horizontal axis and the direction of $x$-half-axis (see Fig. 1). The ellipticity $\tan (\varepsilon)=y / x$. We started with linear polarizations $(\varepsilon=0)$ and then proceeded with elliptical polarizations with three different ellipticities (namely for $\varepsilon=12^{\circ}, 24^{\circ}$, and $\left.36^{\circ}\right)$. The angle $\vartheta$ was, in all the cases, scanned from $0^{\circ}$ to $90^{\circ}$ with the step of $4^{\circ}$. Elliptical polarizations (corresponding to states $\left|\phi_{\mathrm{d}}^{ \pm}\right\rangle$) were produced from horizontal ones by means of a quarter-wave plate rotated by angle $\alpha= \pm \varepsilon$ with respect to the horizontal axis. Then the direction of the $x$-half-axis of the polarization ellipse was tilted by a half-wave plate rotated by $\beta= \pm(\varepsilon+\vartheta) / 2$.

Let us recall that the theoretical probability of successful discrimination of states (7) is

$$
p=2\left(|a|^{2}-|a|^{4}\right) \text {, where }|a|^{2}=x^{2} \cos ^{2} \vartheta+y^{2} \sin ^{2} \vartheta .
$$

Figure 3 shows the coincidence rates measured for linear polarization states. The upper part shows the measurement of Bell states $\left|\Psi^{+}\right\rangle$and $\left|\Psi^{-}\right\rangle$when the data and program states coincide (i.e., the input state is $\left.\left|\phi_{\mathrm{d}}^{+}\right\rangle \otimes\left|\phi_{\mathrm{p}}\right\rangle\right)$, the lower part shows the same measurement in case when they are different (i.e., the input state is $\left.\left|\phi_{\mathrm{d}}^{-}\right\rangle \otimes\left|\phi_{\mathrm{p}}\right\rangle\right)$. Statistical errors are smaller than the symbols of points. Graphs in Fig. 团illustrate the same measurement but with pairs of elliptically polarized states $\left(\varepsilon=24^{\circ}\right.$, i.e., $\left.\tan (\varepsilon)=0.45\right)$.

Throughout the whole paper, $C^{++}$denotes the detection rate of $\left|\Psi^{+}\right\rangle$when the input was in the state $\left|\phi_{\mathrm{d}}^{+}\right\rangle \otimes\left|\phi_{\mathrm{p}}\right\rangle, C^{-+}$denotes the detection rate of $\left|\Psi^{-}\right\rangle$when the input was in the state $\left|\phi_{\mathrm{d}}^{+}\right\rangle \otimes\left|\phi_{\mathrm{p}}\right\rangle, C^{+-}$denotes the detection rate of $\left|\Psi^{+}\right\rangle$when the input was in the state $\left|\phi_{\mathrm{d}}^{-}\right\rangle \otimes\left|\phi_{\mathrm{p}}\right\rangle$, and $C^{--}$denotes the detection rates of $\left|\Psi^{-}\right\rangle$ when the input was in the state $\left|\phi_{\mathrm{d}}^{-}\right\rangle \otimes\left|\phi_{\mathrm{p}}\right\rangle$.

The relative (with respect to all conclusive results) error rates - i.e., fractions of events when we get $\left|\Psi^{+}\right\rangle$ instead of $\left|\Psi^{-}\right\rangle$or viceversa - are about $5 \%$. This value is approximately the same for all measured polarizations. Ideally the error rate should be zero but due to technical imperfections of the setup it gets this small nonzero value.

The main parameter - the probability of success - is plotted in Fig. 5 for four different ellipticities. Symbols denote experimental data, lines represent theoretical predictions (8). The first curve corresponds to linearly polarized photons (acting as data and program qubits), the others, in sequence, to elliptically polarized photons with $\varepsilon=12^{\circ}, 24^{\circ}$, and $36^{\circ}$. As can be seen the data are in a good agreement with the theory.

The probability of success is calculated as an average of normalized detection rates corresponding to successful events (i.e., correct conclusive measurement results) for two possible input states (that are in correspondence with the program state). The averaging takes into account that both the inputs $\left|\phi_{\mathrm{d}}^{+}\right\rangle \otimes\left|\phi_{\mathrm{p}}\right\rangle$ and $\left|\phi_{\mathrm{d}}^{-}\right\rangle \otimes\left|\phi_{\mathrm{p}}\right\rangle$ appear with the same probability $1 / 2$. The "normalization" means that the detection rate is divided by the pairgeneration rate, i.e., by the number of all measurement events per time unit. This quantity is obtained from the coincidence measurement on the shoulder $150 \mu \mathrm{m}$ out of the dip (see Fig. 6). In this situation the two one-photon packets generated by downconversion no longer overlap at the beamsplitter and each of them randomly "decides" whether to go through or to be reflected.

As the splitting ratio of the used beamsplitter is slightly different for vertical and horizontal linear polarizations the normalization measurements were done with $45^{\circ}$ linear polarization states at the inputs. For the set of main measurements (i.e., Bell measurements with balanced arms of Hong-Ou-Mandel interferometer) with the states $\left|\phi_{\mathrm{d}}^{+}\right\rangle \otimes\left|\phi_{\mathrm{p}}\right\rangle$ the input polarization of the normalization measurement were $45^{\circ}, 45^{\circ}$, for the set of main measurements with the states $\left|\phi_{\mathrm{d}}^{-}\right\rangle \otimes\left|\phi_{\mathrm{p}}\right\rangle$ they were $-45^{\circ}$, $45^{\circ}$.

Because our setup and detection electronics are tailored to the measurement of the Bell states $\left|\Psi^{+}\right\rangle$and $\left|\Psi^{-}\right\rangle$we can measure only the events when detectors $D_{1}$ and $\mathrm{D}_{3}$ or $\mathrm{D}_{2}$ and $\mathrm{D}_{4}$ click together or when detectors $\mathrm{D}_{1}$ and $\mathrm{D}_{2}$ or $\mathrm{D}_{3}$ and $\mathrm{D}_{4}$ click in coincidence. For the given $45^{\circ}$ polarizations both these two rates outside the dip should be equal to $1 / 4$ of the pair-generation rate. However, there could be deviations from this $1 / 4$ due to the non-ideal splitting ratio of the beamsplitter. Nevertheless, the sum of these two quantities is immune against this imperfection and proportional to $1 / 2$ of the pairgeneration rate. 


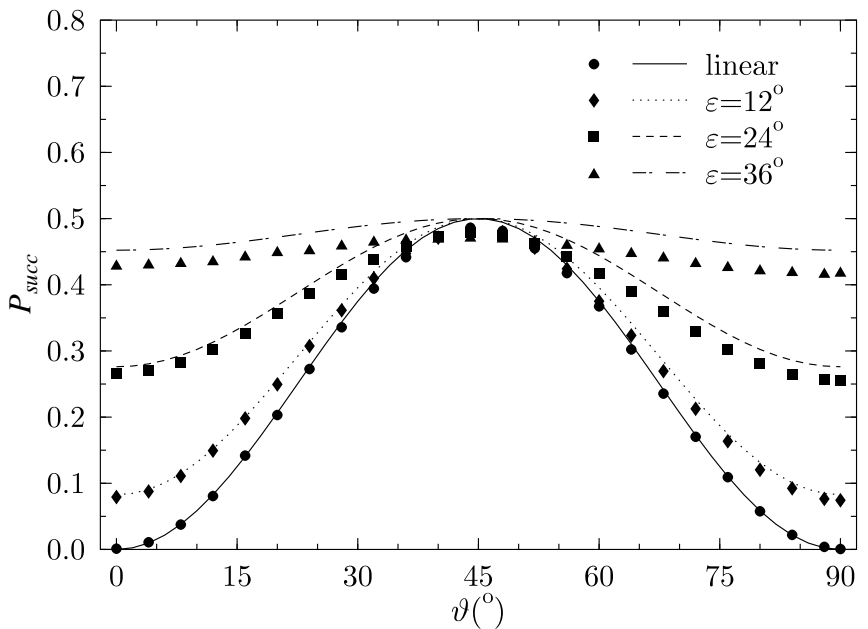

FIG. 5: Probability of successful operation as a function of angle $\vartheta$. Symbols denote experimental data, lines represent theoretical predictions (8). The first curve corresponds to linearly polarized photons, the others, in sequence, to elliptically polarized photons with $\varepsilon=12^{\circ}, 24^{\circ}$, and $36^{\circ}$.

Thus the probability of success is calculated as follows

$$
P_{\text {succ }}=\frac{1}{2}\left[\frac{C^{++}}{2\left(C_{\mathrm{sh}}^{++}+C_{\mathrm{sh}}^{-+}\right)}+\frac{C^{--}}{2\left(C_{\mathrm{sh}}^{--}+C_{\mathrm{sh}}^{+-}\right)}\right],
$$

where $C_{\mathrm{sh}}$ are the above mentioned detection rates measured outside the dip; in particular, $C_{\mathrm{sh}}^{++}$denotes the coincidence rate of $\mathrm{D}_{1}$ and $\mathrm{D}_{3}$ or $\mathrm{D}_{2}$ and $\mathrm{D}_{4}$ with $45^{\circ}$, $45^{\circ}$ polarizations at the inputs, etc. These quantities were always measured just before, or just after the corresponding set of main measurements in order to minimize the influence of the long-term fluctuations (like a laserpower drift etc.). The errors derived from the statistical fluctuations of individual detection rates are smaller than the symbols of points in Fig. 5 The systematic error (the shift of all the data values) is due to non-unit visibility.

\section{PHASE-COVARIANT QUANTUM MULTIMETER}

\section{A. Theory}

Now we will consider a different kind of quantum multimeter. Namely such one that can perform von Neumann measurements on a single qubit in any basis $\left\{\left|\psi_{+}\right\rangle,\left|\psi_{-}\right\rangle\right\}$ located on the equator of the Bloch sphere,

$$
\left|\psi_{ \pm}(\phi)\right\rangle=\frac{1}{\sqrt{2}}\left(|0\rangle \pm e^{i \phi}|1\rangle\right)
$$

where $\phi \in[0,2 \pi)$ is arbitrary. The particular measurement basis is selected by a quantum state of a program register.

It is impossible to perfectly encode such projective measurements into states in finite-dimensional Hilbert

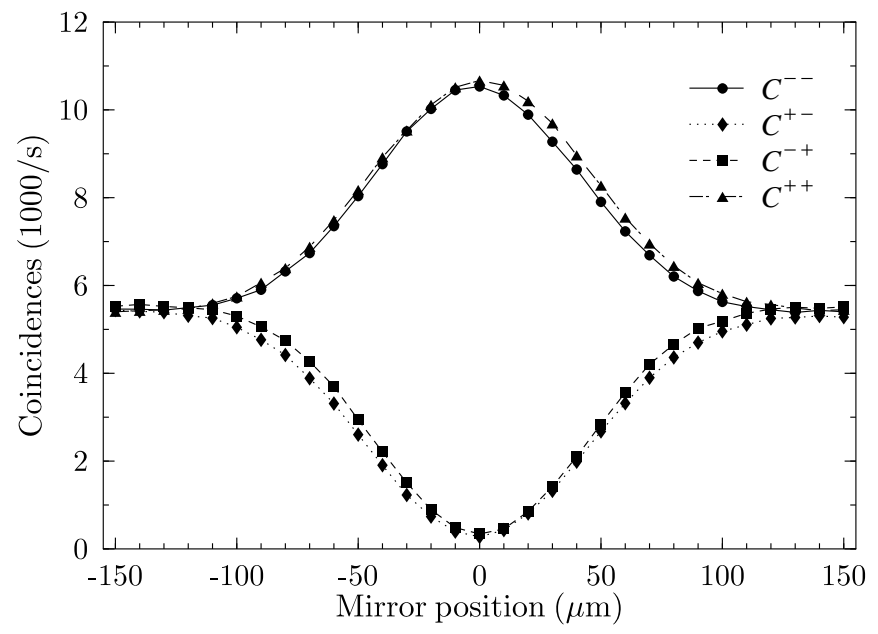

FIG. 6: Detection rates as functions of the mirror position. Here $C^{++}$is a coincidence rate at $\mathrm{D}_{1}$ and $\mathrm{D}_{3}$ or $\mathrm{D}_{2}$ and $\mathrm{D}_{4}$ when the input polarizations were $45^{\circ}, 45^{\circ}$. The quantity $C^{+-}$is a coincidence rate at the same detectors for input polarizations $-45^{\circ}, 45^{\circ}$. Analogously, $C^{-+}$and $C^{--}$are coincidence rates at $\mathrm{D}_{1}$ and $\mathrm{D}_{2}$ or $\mathrm{D}_{3}$ and $\mathrm{D}_{4}$.

space [5, 8]. Nevertheless, it is possible to encode POVMs that represent, in a certain sense, the best approximation of the required projective measurements. A specific way of approximation of a projective measurement is a "probabilistic" measurement that allows for some inconclusive results. In this case, instead of a two-component projective measurement one has a three-component POVM and the third outcome corresponds to an inconclusive result. The optimal multimeter should minimize the error rate at the first two outcomes for a fixed fraction of inconclusive results. As a limit case it is possible to get an error-free operation. Such a multimeter performs the exact projective measurements but with the probability of success lower than one.

The optimal phase-covariant multimeters for the program states $|\Psi\rangle_{\mathrm{p}}$ consisting of $N$ copies of the basis state $\left|\psi_{+}\right\rangle,|\Psi\rangle_{\mathrm{p}}=\left|\psi_{+}\right\rangle^{\otimes N}$ [26], were determined in [8]. For the simplest case $N=1$ that corresponds to a one-qubit program the optimal (fixed) POVM acting on data and program qubits together reads:

$$
\begin{aligned}
\Pi_{ \pm} & =\left|\Psi^{ \pm}\right\rangle\left\langle\Psi^{ \pm}\right|+\frac{1-\eta}{2}\left(\left|\Phi^{+}\right\rangle\left\langle\Phi^{+}|+| \Phi^{-}\right\rangle\left\langle\Phi^{-}\right|\right), \\
\Pi_{?} & =\eta\left(\left|\Phi^{+}\right\rangle\left\langle\Phi^{+}|+| \Phi^{-}\right\rangle\left\langle\Phi^{-}\right|\right),
\end{aligned}
$$

where $\eta \in[0,1]$, and

$$
\begin{aligned}
& \left|\Psi^{ \pm}\right\rangle=\frac{1}{\sqrt{2}}\left(\left|0_{\mathrm{d}}\right\rangle\left|1_{\mathrm{p}}\right\rangle \pm\left|1_{\mathrm{d}}\right\rangle\left|0_{\mathrm{p}}\right\rangle\right), \\
& \left|\Phi^{ \pm}\right\rangle=\frac{1}{\sqrt{2}}\left(\left|0_{\mathrm{d}}\right\rangle\left|0_{\mathrm{p}}\right\rangle \pm\left|1_{\mathrm{d}}\right\rangle\left|1_{\mathrm{p}}\right\rangle\right) .
\end{aligned}
$$

The fidelity of the multimeter can be defined as the probability of a correct measurement outcome in the case of a conclusive result, assuming the data register is prepared in the basis state $\left|\psi_{+}\right\rangle$or $\left|\psi_{-}\right\rangle$with probability $1 / 2$ 
each. The mean fidelity is then obtained by averaging the fidelity over all measurement bases, i.e. over the phase $\phi \in[0,2 \pi)$. The average fidelity of the phase-covariant multimeter depends on the probability of inconclusive result in the following way,

$$
F=\frac{3-2 P_{I}}{4\left(1-P_{I}\right)} .
$$

The effective POVM on the data qubit only (this POVM depends on the program state) is given by

$$
\begin{aligned}
\pi_{ \pm}= & \left(1-P_{I}\right)\left[F\left|\psi_{ \pm}(\phi)\right\rangle\left\langle\psi_{ \pm}(\phi)\right|\right. \\
& \left.+(1-F)\left|\psi_{\mp}(\phi)\right\rangle\left\langle\psi_{\mp}(\phi)\right|\right], \\
\pi_{?}= & P_{I} \mathbb{1} .
\end{aligned}
$$

For $\eta=0$ we have an ambiguous (error-prone) operation with no inconclusive results $\left(P_{I}=0, F=3 / 4\right)$ whilst for $\eta=1$ we get an unambiguous (error-free) but probabilistic measurement device (from time to time we obtain an inconclusive result; $\left.P_{I}=1 / 2, F=1\right)$ 27].

Clearly, when $\eta=1$ then POVM (11) is just a projective measurement on the Bell states $\left|\Psi^{+}\right\rangle,\left|\Psi^{-}\right\rangle$, and on the rest of the four dimensional Hilbert space (that corresponds to inconclusive results). If $\eta<1$ we just "reinterpret" some inconclusive results as "conclusive" ones. I.e., we will treat randomly selected (with probability $1-\eta$ ) inconclusive results as results "+" or "-" (at random). Therefore, it is quite enough to test experimentally only the unambiguous version $(\eta=1)$ of our phase-covariant quantum multimeter as all the other variants $(0 \leq \eta<1)$ can be obtained manipulating the measured data only.

\section{B. Experiment}

The simplest $(N=1)$ phase-covariant multimeter can be experimentally implemented using the same setup as for the programmable quantum-state discriminator (see Fig. 2). The "logical values" 0 and 1 of qubits will be represented by horizontal, $|H\rangle$, and vertical, $|V\rangle$, linearpolarization states of photons, respectively.

The states $\left|\psi_{+}(\phi)\right\rangle$ (10) are prepared from states $|H\rangle$ by two wave plates. The first one is a quarter-wave plate rotated by $\alpha=-\phi / 2$ (with respect to the horizontal axis) and the second one is a half-wave plate rotated by $\beta=\left(90^{\circ}-\phi\right) / 4$. The states $\left|\psi_{-}(\phi)\right\rangle$ are prepared with the wave plates rotated by angles $-\alpha$ and $-\beta$. This corresponds to the shift of $\phi$ by $180^{\circ}$. Detection of $\left|\Psi^{+}\right\rangle$ corresponds to the measurement result connected with the basis state $\left|\psi_{+}(\phi)\right\rangle$, detection of $\left|\Psi^{-}\right\rangle$corresponds to the measurement result connected with the basis state $\left|\psi_{-}(\phi)\right\rangle$. Everything else means an inconclusive result.

\section{Results}

We have measured coincidence rates corresponding to detections of $\left|\Psi^{ \pm}\right\rangle$and calculated probabilities of inconclusive results for phases $\phi$ from $-90^{\circ}$ to $+90^{\circ}$ with step

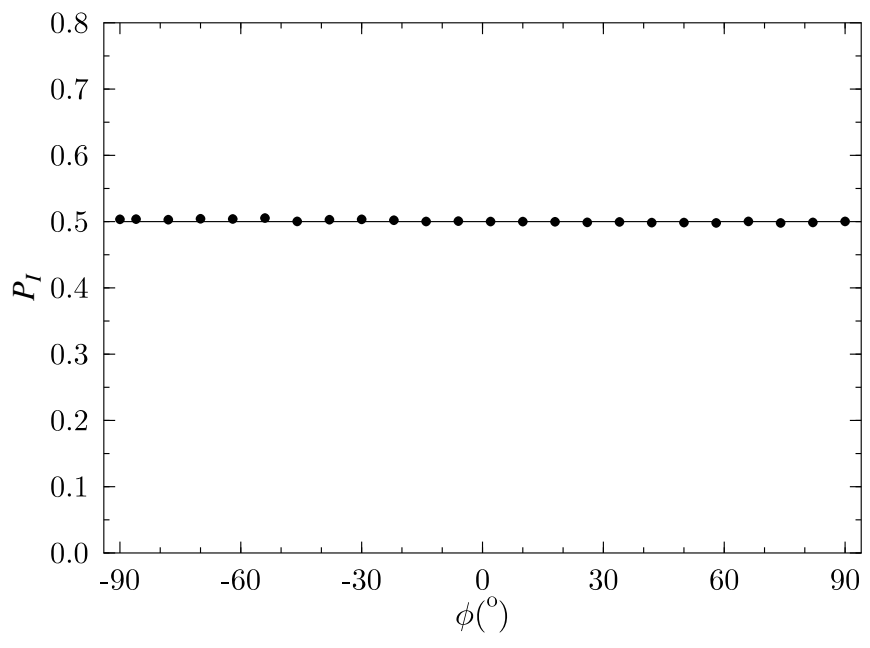

FIG. 7: Probability of inconclusive results for unambiguous operation as a function of phase $\phi$. Symbols denote experimental data, solid line represents the theoretical prediction $P_{I}=1 / 2$.

$8^{\circ}$. The inconclusive-result rate is a "complement" to the rate of "conclusive" results - i.e., both the correct and erroneous ones. Thus

$$
P_{I}=1-\frac{1}{2}\left[\frac{C^{++}+C^{-+}}{2\left(C_{\mathrm{sh}}^{++}+C_{\mathrm{sh}}^{-+}\right)}+\frac{C^{--}+C^{+-}}{2\left(C_{\mathrm{sh}}^{--}+C_{\mathrm{sh}}^{+-}\right)}\right] .
$$

The notation is the same as above.

Graph in Fig. [7 shows the dependence of the probability of inconclusive results (for an unambiguous operation) on phase $\phi$ for input data states $\left|\psi_{+}\right\rangle$and $\left|\psi_{-}\right\rangle$(that coincide with or are orthogonal to the program states, respectively). Symbols denote experimental data, solid line represents the theoretical prediction. The derived errors are smaller than the symbols of points. The experimental data fit well with the calculated value of $P_{I}=1 / 2$. As expected, the probability of inconclusive results does not depend on the measurement basis determined by the phase $\phi$.

The relative error rate (with respect to all conclusive results) - i.e., a fraction of events when we get $\left|\Psi^{+}\right\rangle$instead of $\left|\Psi^{-}\right\rangle$or viceversa - is about $3 \%$. Ideally, in the considered case of the unambiguous operation, the error rate should be zero, but due to technical imperfections of the setup it gets non-zero values.

\section{CONCLUSIONS}

Our experimental results clearly illustrate that the measurement on the data qubit can be quite efficiently controlled by the quantum state of the program qubit (program register). In particular, we emphasize that a classical setting of the angle between the states that shall be unambiguously discriminated and a classical description of the measurement basis in case of projective phasecovariant measurement would require infinitely many bits 
of classical information, while only one quantum bit suffices in the present case to obtain an error-free (although probabilistic) operation.

\section{Acknowledgments}

The authors would like to thank to Martin Hendrych and Miroslav Ježek for their help and advises in the preparatory stage of the experiment. This research was supported under the project LN00A015 of the Ministry of Education of the Czech Republic. JF also acknowledges support from the EU under the project CHIC (IST-2001$32150)$.
[1] C.W. Helstrom, Quantum Detection and Estimation Theory (Academic Press, New York, 1976).

[2] A.S. Holevo, Probabilistic and Quantum Aspects of Quantum Theory (North-Holland, Amsterdam, 1982).

[3] A. Peres, Quantum Theory: Concepts and Methods (Kluwer, Dordrecht, 1993).

[4] M. A. Nielsen and I. L. Chuang, Quantum Computation and Quantum Information (Cambridge Univ. Press, Cambridge, 2000).

[5] M. Dušek and V. Bužek, Phys. Rev. A 66, 022112 (2002).

[6] J. Fiurášek, M. Dušek and R. Filip, Phys. Rev. Lett. 89, 190401 (2002); J. Fiurášek, M. Dušek and R. Filip, Fortschritte der Physik 51, 107 (2003).

[7] M. Hendrych, M Dušek, R Filip, and J Fiurášek, Physics Letters A 310, 95 (2003).

[8] J. Fiurášek and M. Dušek, quant-ph/0308111

[9] J. P. Paz and A. Roncaglia, Phys. Rev. A 68, 052316 (2003).

[10] H. Weinfurter, Europhys. Lett. 25, 559 (1994).

[11] S. L. Braunstein and A. Mann, Phys. Rev. A 51, R1727 (1995).

[12] M. Michler, K. Mattle, H. Weinfurter, and A. Zeilinger, Phys. Rev. A 53, R1209 (1996).

[13] K. Mattle, H. Weinfurter, P. G. Kwiat, and A. Zeilinger, Phys. Rev. Lett. 76, 4656 (1996).

[14] D. Bouwmeester, J. W. Pan, K. Mattle, M. Eibl, H. Weinfurter, and A. Zeilinger, Nature (London) 390, 575
(1997).

[15] A. Chefles, Contemp. Phys. 41, 401 (2001).

[16] S. M. Barnett, Fortschritte der Physik 49, 909 (2001).

[17] I. D. Ivanovic, Phys. Lett. A 123, 257 (1987).

[18] D. Dieks, Phys. Lett. A 126, 303 (1988).

[19] A. Peres, Phys. Lett. A 128, 19 (1988).

[20] G. Jaeger and A. Shimony, Phys. Lett. A 197, 83 (1995).

[21] A. Chefles and S.M. Barnett, Phys. Lett. A 250, 223 (1998).

[22] B. Huttner, A. Muller, J. D. Gautier, H. Zbinden, and N. Gisin, Phys. Rev. A 54, 3783 (1996).

[23] M. Dušek, M. Jahma, N. Lütkenhaus, Phys. Rev. A 62, 022306 (2000).

[24] C. K. Hong, Z. Y. Ou, and L. Mandel, Phys. Rev. Lett. 59, 2044 (1987).

[25] With the same probabilities provided that the frequencies of the occurrence of the input states are also the same.

[26] Since the state $\left|\psi_{-}\right\rangle$can be obtained form $\left|\psi_{+}\right\rangle$via unitary transformation, $\left|\psi_{-}\right\rangle=\sigma_{z}\left|\psi_{+}\right\rangle$where $\sigma_{z}$ denotes the Pauli matrix, all the programs of the form $\left|\psi_{+}\right\rangle^{\otimes j}\left|\psi_{-}\right\rangle^{\otimes N-j}$ are equivalent to the program $\left|\psi_{+}\right\rangle^{N}$.

[27] Notice that an unambiguous phase-covariant multimeter with a single-qubit program represents, in a certain sense, a limit case of the above-discussed programmable discriminator corresponding to the discrimination of two orthogonal states. 